\title{
Patrón alimentario y acceso a los alimentos en familias desplazadas en el municipio de Girón, Santander, Colombia
}

\author{
Gloria Esperanza Prada Gómez, ${ }^{1}$ Oscar Fernando Herrán Falla ${ }^{1}$ \\ y Rosario Ortiz Cárdenas ${ }^{2}$
}

Forma de citar Prada Gómez GE, Herrán Falla OF, Ortiz Cárdenas R. Patrón alimentario y acceso a los alimentos en familias desplazadas en el municipio de Girón, Santander, Colombia. Rev Panam Salud Publica. 2008; 23(4):257-63.

RESUMEN Objetivo. Describir el patrón alimentario y la capacidad de acceso a los alimentos de las familias desplazadas por el conflicto armado en una localidad del departamento de Santander, Colombia.

Métodos. Estudio dietético descriptivo en 101 familias que vivían en condiciones de desplazamiento forzado en la zona urbana y suburbana del municipio de Girón, departamento de Santander, Colombia. Durante el segundo semestre de 2003, el responsable de preparar los alimentos contestó una encuesta sociodemográfica con preguntas sobre el gasto mensual en alimentos, el lugar y la frecuencia de compra, y un recordatorio del consumo de alimentos en las 24 horas previas. El patrón alimentario se estableció según la frecuencia del consumo de alimentos y preparaciones mediante modelos de regresión lineal con el gasto como variable dependiente.

Resultados. De las familias, 34,7\% manifestaron no tener acceso a la compra de alimentos y 13,9\% los recibía como obsequios. El gasto promedio en alimentos fue de 0,52 salarios mínimos vigentes $(S M V)$. La única variable asociada con el gasto fue el número de miembros de la familia que trabajaban y aportaban al presupuesto familiar ( $\mathrm{P}=0,037)$; por cada miembro que trabajaba, el gasto aumentó en 0,07 unidades de SMV (intervalo de confianza de 95\%: 0,004 a 0,149). No se encontró asociación entre el tiempo de desplazamiento y el gasto en alimentos. La calidad de la dieta fue deficiente, ya que no se cumplió la norma recomendada de consumo de frutas, verduras y productos lácteos.

Conclusiones. La inseguridad alimentaria abarcó a 95,0\% de las familias estudiadas aún después de tres años de vivir en condiciones de desplazamiento. La calidad de la dieta era insatisfactoria. Las causas principales de esta situación eran los bajos ingresos familiares y el desconocimiento de alternativas nutricionales más ventajosas. Además de la ayuda alimentaria, los programas de atención a las familias desplazadas deben prever proyectos productivos y de educación nutricional para constituirse en alternativas exitosas.

Palabras clave Conducta alimentaria, seguridad alimentaria, comunidades vulnerables, dieta, Colombia.

1 Universidad Industrial de Santander, Escuela de Nutrición y Dietética, Centro de Investigaciones Epidemiológicas, Observatorio Epidemiológico de Enfermedades Cardiovasculares, Bucaramanga,
Colombia. La correspondencia se debe dirigir a Gloria E. Prada Gómez, Centro de Investigaciones Epidemiológicas, Carrera 32 No. 29-31, tercer piso. Apartado Postal 3E-2021. Bucaramanga, Colom- bia. Correos electrónicos: gprada@uis.edu.co y gprada@telebucaramanga.net.co

2 Empresa Social del Estado Hospital San Juan de Dios, Girón, Santander, Colombia. 
El conflicto armado en Colombia está considerado el de mayor duración en América Latina (1) y es la principal causa de desplazamientos forzados en ese país. Entre 2000 y 2007 se han desplazado 2018511 personas (2), lo que ha generado hacinamiento, desempleo, insatisfacción de las necesidades básicas, alteración del bienestar emocional y una alimentación deficiente en las familias desplazadas.

El deterioro en las condiciones de salud y el bienestar de estas familias es evidente (3). Las malas condiciones de las viviendas, el poco acceso a los servicios públicos (solo $44,0 \%$ de los hogares desplazados disponen de servicios de acueducto) y la inadecuada manipulación de los residuos aumentan el riesgo de enfermar; la quinta parte de la población desplazada no tiene acceso a los servicios de salud (4).

El desplazamiento es un factor de vulnerabilidad que provoca la inseguridad alimentaria debido a los cambios inesperados que afectan a las condiciones de vida. Diversos estudios muestran que el consumo de energía de las familias desplazadas es deficiente y su patrón alimentario sufre modificaciones. Por ejemplo, en las familias rurales se sustituye el consumo de algunos alimentos que antes producían - como frutas, leche y huevos- por carbohidratos (1), lo que influye negativamente en la calidad de la dieta y el estado nutricional de los niños y los adultos. En el caso de la población infantil, la desnutrición aguda es cuatro veces mayor que la media del país y la crónica, dos veces mayor (4).

Como respuesta gubernamental a esta situación, en 1995 se creó en Colombia el Programa de Atención al Desplazamiento y diez años después, el Ministerio del Interior y de Justicia adoptó el Plan Nacional para la Atención Integral a la Población Desplazada por la Violencia, mediante el decreto 250 del 7 de febrero de 2005 (5), que abarcaba la atención a la seguridad alimentaria de esta población. Siguiendo las recomendaciones planteadas por expertos en nutrición $(6,7)$, este plan prevé que todos los programas se basen en los patrones socioculturales de la población blanco, con vista a lograr un mayor impacto.
En Colombia hay pocos estudios sobre la seguridad alimentaria y las condiciones de acceso a los alimentos en familias desplazadas que permitan establecer una línea de base para diseñar y evaluar las intervenciones. El objetivo de este estudio fue describir el patrón alimentario y la capacidad de acceso a los alimentos de las familias desplazadas por el conflicto armado en una localidad del departamento de Santander, Colombia. Esta información ayudará a orientar las acciones que en materia de seguridad alimentaria tiene contempladas el Plan Nacional para la Atención Integral a la Población Desplazada por la Violencia.

\section{MATERIALES Y MÉTODOS}

Se realizó un estudio dietético descriptivo que abarcó las 101 familias que vivían en condiciones de desplazamiento forzado en la zona urbana y suburbana del municipio de Girón, departamento de Santander, Colombia, según el Registro Único de Población Desplazada, de la Agencia Presidencial para la Acción Social y la Cooperación Internacional. Estas familias eran beneficiarias de un programa de ayuda alimentaria.

Al responsable de la preparación de los alimentos en cada hogar se le aplicó una encuesta sociodemográfica mediante entrevista directa, seguida de un recordatorio del consumo de alimentos en las 24 horas previas, en el que se especificó el alimento o preparación y la cantidad consumida. Además, se preguntó el gasto mensual en alimentos, el lugar y la frecuencia de compra de cada grupo de alimentos (perecederos y no perecederos). El gasto mensual en alimentos de calculó en unidades de salario mínimo legal vigente (SMLV).

Las encuestas se aplicaron en el segundo semestre de 2003 por estudiantes de la Escuela de Nutrición y Dietética de la Universidad Industrial de Santander, entrenados en la aplicación de métodos de recolección de datos con recordatorio (8). La codificación de los alimentos se realizó según tablas de alimentos y preparaciones específi- cas para esta región del país (9), bajo la supervisión de los investigadores principales.

El patrón alimentario se estableció según la frecuencia del consumo de alimentos y preparaciones. La dieta se evaluó mediante los parámetros recomendados por el Instituto Colombiano de Bienestar Familiar, entidad adscrita al Ministerio de la Protección Social dedicada a la protección integral de la familia y la niñez mediante la administración del Sistema Nacional de Bienestar Familiar (10). Las variables sociodemográficas y el gasto en alimentos se compararon según la zona de procedencia del desplazamiento (urbano o rural) mediante la prueba de la $t$ de Student (variables continuas) y de la ji al cuadrado (variables categóricas). Debido a la evidente asimetría del gasto en alimentos, se empleó la mediana para describir esta variable. A fin de explorar la asociación con las variables estudiadas se elaboraron modelos de regresión lineal con el gasto como variable dependiente; en todos los casos se comprobó la distribución normal de los datos y otros requisitos básicos de linealidad (11).

Todos los registros se digitaron por duplicado y se validaron en Epi Info v. $6.04 \mathrm{~d}$ (12). Se determinó el patrón alimentario mediante el sistema de información para cuantificar la ingesta SICI v. 2.0 (13). El procesamiento de datos y los cálculos estadísticos se realizaron con el programa Stata/SE v. 8.2 (14).

Todos los participantes firmaron un documento de consentimiento informado. El estudio recibió la aprobación del Comité de Ética en Investigación de la Facultad de Salud de la Universidad Industrial de Santander.

\section{RESULTADOS}

En promedio, las 101 familias estudiadas estaban compuesta por 6,2 miembros, de los cuales 1,6 aportaban al gasto familiar. Casi la tercera parte de las personas que componían estas familias eran menores de 7 años de edad. En general, 34,7\% de las familias manifestaron no tener acceso a la compra de alimentos y 13,9\% del total los 
CUADRO 1. Características de las familias desplazadas estudiadas, Girón, Santander, Colombia, 2003

\begin{tabular}{|c|c|c|c|c|c|c|c|}
\hline \multirow[b]{2}{*}{ Variable } & \multicolumn{2}{|c|}{$\begin{array}{c}\text { Total } \\
n=101\end{array}$} & \multicolumn{2}{|c|}{$\begin{array}{l}\text { Procedencia urbana } \\
\qquad n=60\end{array}$} & \multicolumn{2}{|c|}{$\begin{array}{l}\text { Procedencia rural } \\
\qquad n=41\end{array}$} & \multirow[b]{2}{*}{$P^{\mathrm{b}}$} \\
\hline & $\begin{array}{l}\text { Valor } \\
(\%)\end{array}$ & IC $95 \% a$ & $\begin{array}{l}\text { Valor } \\
(\%)\end{array}$ & $\mathrm{IC} 95 \%$ & $\begin{array}{l}\text { Valor } \\
(\%)\end{array}$ & IC95\% & \\
\hline Edad de la madre, años & 29,0 & $27,2-30,8$ & 29,0 & $26,6-31,4$ & 28,9 & $26,0-31,9$ & 0,972 \\
\hline Tiempo como desplazados, meses & 31,3 & $26,5-36,1$ & 27,7 & $21,8-33,5$ & 37,6 & $29,3-45,9$ & 0,046 \\
\hline Consumo de la madre, kilocalorías & 1511 & $1404-1617$ & 1520 & $1387-1654$ & 1497 & $1315-1680$ & 0,833 \\
\hline Número de miembros en la familia & 6,2 & $5,7-6,6$ & 5,8 & $5,3-6,3$ & 6,7 & $5,9-7,5$ & 0,039 \\
\hline Menores de 7 años & 1,9 & $1,7-2,1$ & 1,9 & $1,6-2,1$ & 2,0 & $1,7-2,3$ & 0,493 \\
\hline Trabajan y aportan & 1,6 & $1,4-1,7$ & 1,4 & $1,2-1,6$ & 1,8 & $1,5-2,2$ & 0,012 \\
\hline Come fuera del hogar & 0,31 & $0,20-0,42$ & 0,35 & $0,20-0,50$ & 0,24 & $0,07-0,41$ & 0,353 \\
\hline Gasto en alimentos, SMLV/mes ${ }^{c}$ & 0,53 & $0,47-0,58$ & 0,53 & $0,45-0,61$ & 0,52 & $0,44-0,61$ & 0,911 \\
\hline Gasto en alimentos perecederos, SMLV/mes & 0,26 & $0,22-0,30$ & 0,26 & $0,22-0,31$ & 0,30 & $0,25-0,35$ & 0,486 \\
\hline Recibe alimentos obsequiados & $14(13,9)$ & & $8(13,3)$ & & $6(14,6)$ & & 0,853 \\
\hline Cuenta con dinero para alimentos & $66(65,3)$ & & $38(63,3)$ & & $28(68,3)$ & & 0,607 \\
\hline Jefe del hogar & & & & & & & 0,981 \\
\hline Madre & $58(57,4)$ & & $34(56,7)$ & & $24(58,5)$ & & \\
\hline Padre & $23(22,8)$ & & $14(23,3)$ & & $9(22,0)$ & & \\
\hline Abuela u otros & $20(19,8)$ & & $12(20,0)$ & & $8(19,5)$ & & \\
\hline
\end{tabular}

a Intervalo de confianza de 95,0\%.

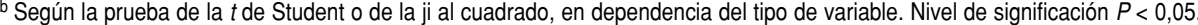

c SMLV: unidades de salario mínimo legal vigente. El salario mínimo legal vigente mensual en 2003 era de $\$ 332000,00$ pesos colombianos (US $\$ 1,00=\$ 2877,00)$.

CUADRO 2. Lugar, frecuencia de compra y gasto en alimentos perecederos y no perecederos en familias desplazadas, Girón, Santander, Colombia, 2003

\begin{tabular}{|c|c|c|c|c|c|c|}
\hline \multirow[b]{3}{*}{ Lugar de compra } & \multicolumn{6}{|c|}{ Frecuencia de compra } \\
\hline & \multicolumn{2}{|c|}{ Diario } & \multicolumn{2}{|c|}{ Semanal } & \multicolumn{2}{|c|}{ Quincenal o mayor } \\
\hline & $\begin{array}{l}\text { No. de } \\
\text { familias }\end{array}$ & $\begin{array}{l}\text { Mediana del } \\
\text { gasto } \pm \mathrm{DE}^{\mathrm{b}}\end{array}$ & $\begin{array}{l}\text { No. de } \\
\text { familias }\end{array}$ & $\begin{array}{l}\text { Mediana del } \\
\text { gasto } \pm \mathrm{DE}\end{array}$ & $\begin{array}{l}\text { No. de } \\
\text { familias }\end{array}$ & $\begin{array}{c}\text { Mediana del } \\
\text { gasto } \pm \mathrm{DE}\end{array}$ \\
\hline \multicolumn{7}{|l|}{ Alimentos perecederos } \\
\hline Micro expendio local (tienda) & 36 & $0,8 \pm 0,7$ & 8 & $1,7 \pm 1,0$ & 3 & $6,6 \pm 2,8$ \\
\hline Plaza de mercado & 12 & $1,3 \pm 0,6$ & 15 & $3,9 \pm 2,0$ & 5 & $3,9 \pm 4,2$ \\
\hline Camión (ambulante) & 2 & $1,4 \pm 0,6$ & 4 & $2,0 \pm 1,2$ & 4 & $1,4 \pm 1,3$ \\
\hline Supermercado & 2 & $4,2 \pm 1,5$ & 2 & $13,5 \pm 17,9$ & $N^{c}$ & ND \\
\hline \multicolumn{7}{|l|}{ Alimentos no perecederos } \\
\hline Micro expendio local (tienda) & 52 & $0,8 \pm 0,8$ & 9 & $6,5 \pm 4,6$ & ND & ND \\
\hline Plaza de mercado & 2 & $1,3 \pm 0,0$ & 5 & $7,9 \pm 2,3$ & 3 & $7,9 \pm 4,0$ \\
\hline Supermercado & 9 & $7,9 \pm 3,6$ & 7 & $7,9 \pm 2,2$ & 4 & $19,6 \pm 15,0$ \\
\hline
\end{tabular}

recibía como obsequios. En $57,4 \%$ de las familias, la madre era la jefa del hogar y $0,31 \%$ de los miembros de las familias estudiadas consumían alimentos fuera del hogar, principalmente el padre $(30,0 \%$ de ellos). Predominaron los desplazados provenientes del propio departamento de Santander $(54,5 \%$ de las familias), seguidos de los procedentes de los departamentos limítrofes de César $(19,4 \%)$, Norte de Santander $(12,8 \%)$ y Bolívar $(9,6 \%)$ (cuadro 1$)$.
Independientemente de si los alimentos eran perecederos o no, los microexpendios locales de alimentos (tiendas) constituían el principal sitio de abastecimiento y $58,6 \%$ de las familias se abastecían en ellos; 22,8\% lo hacía en los centros generales de abastecimiento de alimentos (plazas de mercado) y solo $13,0 \%$ compraba sus alimentos en supermercados. La mayoría de las familias hacía sus compras diariamente y casi la mitad de la in- versión en alimentos se destinaba a productos frescos o perecederos.

En el cuadro 2 se describe el gasto en alimentos en unidades de $\mathrm{SMLV}^{3}$, según la frecuencia y el lugar de compra. En promedio, cada familia gastaba 0,52 SMLV en alimentos. Según el mo-

\footnotetext{
El SMLV mensual en 2003 era de $\$ 332000$ pesos colombianos (US\$1,00 = \$2 877 pesos colombianos, según la tasa de cambio oficial en Colombia en 2003. Banco de la Republica, Colombia, 2003[15]).
} 
CUADRO 3. Consumo de alimentos básicos por familias desplazadas, según el recordatorio de las 24 horas previas, Girón, Santander, Colombia, 2003

\begin{tabular}{|c|c|}
\hline Alimentos & Frecuencia de consumo, (\%) \\
\hline Cebolla común & $101(100)$ \\
\hline Arroz & $93(92,1)$ \\
\hline Aceites vegetales & $92(91,1)$ \\
\hline Panela (derivado de la caña de azúcar) & $88(87,1)$ \\
\hline Tomate & $85(84,2)$ \\
\hline Рapa & $84(83,2)$ \\
\hline Leche entera pasteurizada & $60(59,4)$ \\
\hline Azúcar & $60(59,4)$ \\
\hline Huevo & $56(55,5)$ \\
\hline Limón & $53(52,5)$ \\
\hline Pan & $52(51,5)$ \\
\hline Harina de maíz & $52(51,5)$ \\
\hline Tostado o calado (pan a base de harina de trigo) & $49(48,5)$ \\
\hline Plátano hartón & $47(46,5)$ \\
\hline Caldo de gallina deshidratado & $45(44,6)$ \\
\hline Cilantro & $43(42,6)$ \\
\hline Infusión caliente de café & $40(39,6)$ \\
\hline Pastas & $38(37,6)$ \\
\hline Cebolla cabezona & $38(37,6)$ \\
\hline Zanahoria & $36(35,6)$ \\
\hline Yuca blanca & $33(32,7)$ \\
\hline Ajo & $30(29,7)$ \\
\hline Hueso carnudo & $27(26,7)$ \\
\hline Achiote, materia colorante & $27(26,7)$ \\
\hline Mantequilla & $24(23,8)$ \\
\hline Carne de bovino & $20(19,8)$ \\
\hline Bebida caliente a base de chocolate & $20(19,8)$ \\
\hline Habichuela & $19(18,8)$ \\
\hline Saborizantes & $18(17,8)$ \\
\hline Auyama & $18(17,8)$ \\
\hline Arveja verde & $15(14,9)$ \\
\hline Pollo & $12(11,9)$ \\
\hline Lenteja & $11(10,9)$ \\
\hline Arracacha amarilla & $11(10,9)$ \\
\hline Manteca & $9(8,9)$ \\
\hline Arveja seca & $9(8,9)$ \\
\hline Frijol & $9(8,9)$ \\
\hline Bienestarina (mezcla vegetal) & $7(6,9)$ \\
\hline Queso & $7(6,9)$ \\
\hline Gaseosa (bebida azucarada carbonatada) & $5(5,0)$ \\
\hline
\end{tabular}

delo de regresión lineal, la única variable asociada con el gasto total en alimentos fue el número de miembros de la familia que trabajaban y aportaban al presupuesto familiar $(P=0,037)$, de manera que por cada miembro de la familia que trabajaba aumentó el gasto en 0,07 unidades de SMLV (intervalo de confianza de 95\%: 0,004 a 0,149). Sin embargo, la capacidad explicativa del modelo fue baja (5,0\%). No se encontró asociación alguna entre el tiempo vivido en condiciones de desplazamiento, el gasto en alimentos y otras variables estudiadas. mayor consumo fue el limón; solamente $35,6 \%$ de las familias consumía zanahoria.

En cuanto al patrón alimentario de cada comida, casi en la mitad de los hogares se consumía caldo de papa (sopa de papa en agua con sal y leche) en el desayuno, en $41,6 \%$ se consumía agua de panela (infusión caliente preparada con un derivado sólido de la caña de azúcar, la panela) y en $38,6 \%$, arepas (tortillas preparada con harina de maíz) (cuadro 4). El arroz era la principal preparación del almuerzo y lo consumía $83,2 \%$ de las familias; $61,4 \%$ consumía limonada o jugo de frutas, 19,8\% comía algún tipo de carne y solamente $10,9 \%$ consumía verduras en ensalada. La última comida del día estaba constituida principalmente por agua de panela, arroz y tubérculos. De esta forma, las leguminosas, las frutas y las verduras se consumían casi exclusivamente en el almuerzo. En todas las comidas se tomaba alguna bebida.

La calidad de la dieta fue deficiente, ya que no se cumplió la norma recomendada por el Instituto Colombiano de Bienestar Familiar, tanto con respecto a las frutas y las verduras como a los productos lácteos. Solo $50,0 \%$ de las familias consumía productos lácteos, siempre en pequeñas cantidades (no más de $25 \%$ del consumo mínimo recomendado). Las hortalizas, verduras y leguminosas verdes satisfacían solo $15,0 \%$ del consumo recomendado, mientras que las frutas cubrían solamente el 12,5\% (cuadro 5).

\section{DISCUSIÓN}

Más de $80,0 \%$ de las familias utililas preparaciones, como cebolla, aceite y tomate; otros alimentos importantes dentro de la canasta alimentaria, fueron los cereales, los tubérculos, las raíces y los plátanos (cuadro 3).

Las principales fuentes de proteína animal eran la leche y el huevo, consumidos por más de la mitad de las familias; menos de la tercera parte de las familias consumía algún tipo de carne y $28,7 \%$ del total de las familias consumía proteínas vegetales a partir de lenteja, arveja seca y fríjol. La fruta de
En el conjunto de familias estudiadas, $57,4 \%$ de los hogares estaba a cargo de la madre, cifra superior al valor de 55,0\% encontrado en otro estudio realizado en Colombia en seis ciudades receptoras de familias desplazadas (4). Esta situación compromete la seguridad alimentaria de las familias, ya que los ingresos de las mujeres son por lo general inferiores al de los hombres, tanto antes como después del desplazamiento. Según estudios realizados por el Programa Mun- 
CUADRO 4. Preparaciones y alimentos consumidos en cada comida por familias desplazadas, según el recordatorio de las 24 horas previas, Girón, Santander, Colombia, 2003

\begin{tabular}{llc}
\hline Comida & \multicolumn{1}{c}{ Preparación } & Frecuencia de uso (\%) \\
\hline Desayuno & & \\
& Caldo de papa $^{\mathrm{a}}$ & $48(47,5)$ \\
& Agua de panela $^{\mathrm{b}}$ & $42(41,6)$ \\
& Arepas & $39(38,6)$ \\
& Pan & $33(32,7)$ \\
& Huevos & $30(29,7)$ \\
& Infusión de café con leche & $18(17,8)$ \\
Almuerzo & Infusión caliente de café & $13(12,9)$ \\
& & \\
& Arroz & $84(83,2)$ \\
& Limonada o jugo de frutas & $62(61,4)$ \\
& Papa, yuca o plátano cocido & $44(43,6)$ \\
& Guiso de leguminosas & $26(25,7)$ \\
& Saborizantes & $17(16,8)$ \\
& Carne de bovino guisada & $13(12,9)$ \\
& Huevos & $12(11,9)$ \\
Ensalada de verduras & $11(10,9)$ \\
Comida & $7(6,9)$ \\
& Agua de panela & $7(6,9)$ \\
& Agua de panela & \\
& Arroz seco & $40(39,6)$ \\
& Papa, yuca o plátano & $26(25,7)$ \\
& Arepas & $23(22,8)$ \\
Huevos & $21(20,8)$ \\
Pan & $20(19,8)$ \\
Café con leche & $19(18,8)$ \\
Bebida caliente a base de chocolate & $17(16,8)$ \\
Caldo de papa & $9(8,9)$ \\
Pastas guisadas & $6(5,9)$ \\
& & $6(5,9)$ \\
\hline
\end{tabular}

a Sopa de papa en agua con sal y leche.

b Infusión caliente preparada con un derivado sólido de la caña de azúcar, la panela.

${ }^{c}$ Tortillas preparada con harina de maíz.

CUADRO 5. Consumo diario de alimentos en familias desplazadas, según el recordatorio de las 24 horas previas, Girón, Santander, Colombia, 2003

\begin{tabular}{|c|c|c|c|}
\hline Grupo de alimento & $\begin{array}{l}\text { Consumo mínimo } \\
\text { recomendado }\end{array}$ & $\begin{array}{l}\text { Consumo } \\
\text { estimado }\end{array}$ & $\begin{array}{l}\text { Porcentaje del } \\
\text { consumo mínimo } \\
\text { recomendado }\end{array}$ \\
\hline Cereales, raíces, tubérculos y plátanos & 4,5 & 5,0 & 111,1 \\
\hline Hortalizas, verduras y leguminosas verdes ${ }^{b}$ & 2,0 & 0,3 & 15,0 \\
\hline Frutas & 4,0 & 0,5 & 12,5 \\
\hline Carnes, huevos, leguminosas secas & 1,0 & 2,0 & 200,0 \\
\hline Productos lácteos & 2,0 & 0,5 & 25,0 \\
\hline Grasas $^{\mathrm{C}}$ & 6,0 & 6,0 & 100,0 \\
\hline Azúcares y dulces & 4,0 & 4,0 & 100,0 \\
\hline
\end{tabular}

a Porciones de intercambio recomendadas para un día en escolares, adolescentes y adultos (10).

b Solo cuando constituyen el ingrediente principal.

c El cálculo de la grasa abarca la utilizada como medio de cocción.

dial de Alimentos de las Naciones Unidas, las familias encabezadas por mujeres constituyen un grupo altamente vulnerable y su consumo alimentario diario promedio es de $1755 \mathrm{kcal}$, mientras que el consumo promedio diario en las familias que cuentan con ambos padres es de $2030 \mathrm{kcal}$ (1).

El concepto de seguridad alimentaria en el hogar hace referencia al acceso a "una canasta de alimentos nutricionalmente adecuada, segura y culturalmente aceptable" (16). La canasta básica alimentaria se define como el conjunto de alimentos representativos del consumo poblacional en cantidades suficientes para cubrir la necesidad de energía de una familia promedio (17). Sin embargo, en los países en desarrollo, la seguridad alimentaria se ve afectada por las restricciones económicas originadas por los bajos ingresos familiares que limitan la compra y el consumo de alimentos (18).

Los resultados obtenidos demuestran que las familias estudiadas gastaban en alimentos 0,52 del SMLV en promedio, lo que les permitiría cubrir sólo $44,0 \%$ del costo total de la canasta básica alimentaria (19). De las familias estudiadas, 95,0\% no podía cubrir los costos de esa canasta. Estos resultados demuestran que existe una brecha entre las familias desplazadas y otras también consideradas pobres que no viven en condiciones de desplazamiento, ya que mientras $45,0 \%$ de los hogares pobres de la misma zona geográfica no podía cubrir los gastos de la canasta básica alimentaria, este porcentaje aumentó a más del doble en las familias desplazadas (19). La inversión en alimentos en hogares de otros municipios del departamento de Santander es casi tres veces mayor que la observada en las familias desplazadas (20).

Este estudio mostró la importancia de mejorar la oferta de trabajo a la población desplazada, ya que por cada miembro de la familia que trabajaba, el gasto en alimentos aumentó en 7,0\% del SMLV (15,0\% del gasto real), lo que se traduce en un mayor acceso a los alimentos y una mayor seguridad alimentaria.

La proporción de familias desplazadas que compran sus alimentos perecederos en pequeñas tiendas locales $(50,0 \%)$ es mucho mayor que la encontrada en otros estudios realizados en este departamento (11,0\%). Esto puede deberse a que en estos expendios, los alimentos se venden en cantidades acordes a la capacidad de compra de la población (por ejemplo, tres cucharadas de aceite, una onza de arroz, 
veinte habichuelas) (21). Estudios nacionales muestran, además, que la gran mayoría de los desplazados recurren a las pequeñas tiendas locales por la cercanía al hogar y el crédito que otorgan para la compra de alimentos (1). La frecuencia de compra diaria que predominó refleja que la expectativa de empleo no supera el día presente.

Los resultados obtenidos revelan que el patrón alimentario de las familias desplazadas difiere del de otros grupos vulnerables no desplazados: las familias estudiadas consumían el doble de harina de maíz y hasta 30,0\% más de arroz y panela que otros grupos (22-25). Estas familias consumían casi tres veces más plátanos que lo observado en otros estudios, tal vez debido a su bajo costo o a que estas personas provenían de zonas donde el plátano es un alimento básico. Llama la atención el elevado uso de saborizantes y colorantes (como el azúcar en polvo con sabores artificiales que se utiliza para preparar bebidas refrescantes), ya que esta práctica podría sustituirse por el consumo de jugos de frutas, mucho más nutritivos.

En cuanto a las fuentes proteicas, sólo $31,7 \%$ de las familias consumió carne de vacuno o pollo el día antes de la encuesta y 59,4\% leche, menos de lo observado en otros grupos vulnerables no desplazados $(56,3 \%$ y $70,2 \%$, respectivamente) (22). Por lo visto, en esta población se sustituyó la carne por huevos y leguminosas, similar a lo informado por otros autores locales (23); esto puede explicar que el consumo de huevos fuera aproximadamente el doble que el informado en otros estu$\operatorname{dios}(20,24)$.

Desde el punto de vista nutricional, el patrón alimentario de esta población puede considerarse desventajoso por el poco aporte de proteínas de alto valor nutricional, hierro, vitaminas en general y del complejo B en particular, fibras, antioxidantes y nutrientes vegetales. Además, la grasa consumida era predominantemente de origen animal (manteca), posiblemente por su menor costo en comparación con la vegetal, lo que favorece el desarrollo de algunas enfermedades crónicas, como el cáncer de mama, de próstata y de colon y la enfermedad cardiovascular (26-28).

Como factor positivo se debe resaltar el elevado consumo de leguminosas secas, que aunque son de bajo costo tienen un alto contenido de ácido fólico, de gran importancia y deficitario en la población colombiana $(29,30)$.

Estos resultados deben tomarse en cuenta para diseñar intervenciones nutricionales dirigidas a esta población, con miras a mejorar la eficiencia del gasto en alimentos y la calidad de la dieta.

La limitación más importante de este estudio es que se realizó un solo recordatorio del consumo de alimentos en las veinticuatro horas previas, lo que puede haber introducido un sesgo de información al no permitir captar la variabilidad de la dieta entre diferentes días (31). Otro posible sesgo de información tiene que ver con el gasto en alimentos que declararon, ya que este tipo de población puede tener la tendencia a declararse en peores condi- ciones socioeconómicas que las reales para ingresar a programas de ayuda estatal. En la presente investigación no se pudo comprobar la veracidad de la información recibida.

Se puede concluir que la inseguridad alimentaria abarcaba a 95,0\% de las familias estudiadas y que, en general, la calidad de la dieta era insatisfactoria. Las causas principales de esta situación eran los bajos ingresos familiares -asociados con el limitado número de personas que trabajaban y aportaban a la economía familiar - y el desconocimiento de alternativas nutricionales más ventajosas. Estos resultados indican que después de tres años de vivir en condiciones de desplazamiento, las familias estudiadas no habían resuelto su inseguridad alimentaria.

Además de la ayuda alimentaria, los programas de atención a las familias desplazadas deben prever proyectos productivos y de educación nutricional para constituirse en alternativas exitosas.

Agradecimientos. Los autores agradecen al Instituto Colombiano de Bienestar Familiar y al Programa Mundial de Alimentos de las Naciones Unidas por el apoyo logístico; a la Empresa Social del Estado (ESE) Hospital San Juan de Dios, de Girón, Santander, Colombia y la Universidad Industrial de Santander (UIS), de Santander, Colombia, por la cofinanciación de este proyecto; y a los estudiantes de último año de la Escuela de Nutrición y Dietética de la UIS, por su dedicación durante al estudio.

\section{REFERENCIAS}

1. Programa Mundial de Alimentos. Estudio de caso de las necesidades alimentarias de la población desplazada de Colombia. Santa Fe de Bogotá: PMA; 2001. Hallado en http:// www.disaster-info.net/desplazados / informes/pma/FinalReportSpanish.htm. Acceso el 1 de febrero de 2008.

2. República de Colombia, Red de Solidaridad Social. Sistema Único de Registro. Índice General de Tabulados de Población Desplazada. Santa Fe de Bogotá: Agencia Presidencial para la Acción Social y la Cooperación Internacional; 2007. Hallado en http:/ /www.accion social.gov.co/
Estadisticas/publicacion $\% 20$ dic $\% 2031 \% 20 \mathrm{de} \%$ 202007.htm. Acceso el 2 de febrero de 2007.

3. Millán NH, Ochoa LA. Papel de la ayuda alimentaria en la garantía de la seguridad alimentaria de la población en situación de desplazamiento forzoso [tesis para optar el grado de Nutricionista Dietista]. Santa Fe de Bogotá: Universidad Nacional de Colombia; 2003.

4. Organización Panamericana de la Salud, Universidad de Antioquia. Comparación de la situación de salud, entre población en situación de desplazamiento y receptora, en seis ciudades, 2002-2003. Santa Fe de Bogotá: OPS;
2004. (Serie Salud y Desplazamiento en Colombia, Modulo 1).

5. República de Colombia, Ministerio del Interior y Justicia. Plan Nacional para la Atención Integral a la Población Desplazada por la Violencia. Decreto número 250 de febrero 7 de 2005. Santa Fe de Bogotá: Presidencia de la República; 2005. Hallado en http://www. disaster-info.net/desplazados/legislacion/ Decreto_250_07022005.pdf. Acceso el 1 de febrero de 2008 .

6. Organización de las Naciones Unidas para la Agricultura y la Alimentación. Guía metodo- 
lógica de comunicación social en nutrición. Roma: FAO; 1996

7. Morón C. Guía para la gestión municipal de programas de seguridad alimentaria y nutrición. Santiago: FAO, Oficina Regional para América Latina y el Caribe; 2001.

8. Buzzard M. 24-hour dietary recall and food record methods. En: Willett WC, ed. Nutritional epidemiology. New York: Oxford University Press; 1998. Pp. 50-73.

9. Herrán OF, Bautista LE, Quintero DC. Tabla de composición de alimentos consumidos en Bucaramanga. 2. ${ }^{a}$ ed. Bucaramanga, Colombia: Centro de Investigaciones Epidemiológicas, Universidad Industrial de Santander; 2003.

10. República de Colombia, Instituto Colombiano de Bienestar Familiar, Fundación Colombiana para la Nutrición Infantil. Consumo de alimentos en Colombia. 1998. Guías alimentarias para la población Colombiana mayor de dos años. Caracterización de la población. Bogotá: ICBF; 2000.

11. Hamilton LC. Regression with graphics. Belmont, California: Duxbury Press; 1991. Pp. 65-108.

12. Centers for Desease Control and Prevention. Epi Info, versión 6.04d. Atlanta, Georgia: CDC; 2001.

13. Prada GE. Sistema de información para cuantificar la ingesta, SICI, versión 2.0. Bucaramanga, Colombia: Universidad Industrial de Santander; 2001

14. StataCorp. Stata Statistical Sofware, release 8.2. College Station, Texas: Stata Corporation; 2003.

15. República de Colombia, Banco de la República. Tasa de cambio. Disponible en: http:// www.banrep.gov.co/estad/dsbb/sec_ext_ 011.xls. Acceso el 1 de febrero de 2007.

16. Eide A, Oshaug A, Eide WB. Food security and the right to food in international law and development. Transnatl Law Contemp Probl. 1991;1(2):416-67.

17. Crovetto M. La canasta básica de alimentos en la política alimentaria y nutricional de Chile [tesis para optar al grado de maestro en Planificación y Alimentación]. Santiago: Universidad de Chile; 1993.

18. Torres F, Trápaga Y, Delgadillo J, Oceguera D, Zamora J, García JC, et al. La alimentación de los mexicanos en la alborada del tercer milenio. México, D.F.: Instituto de Investigaciones Económicas, UNAM; 2004.

19. Herrán OF, Prada GE, Patiño GA. Canasta básica alimentaria e índice de precios en Santander, Colombia, 1999-2000. Salud Publica Mex. 2003:45:35-42.

20. Prada GE, Álvarez A. Caracterización de la situación alimentaria en Santander: Vélez, Charalá y Floridablanca. Informe final de investigación. Bucaramanga, Colombia: Secretaría de Salud de Santander, Universidad Industrial de Santander; 2002.

21. Schejtman A. Economía política de los sistemas alimentarios en América Latina. Santiago: FAO; 1994.

22. Herrán OF, Prada GE. Situación alimentaria en mujeres de dos municipios Santandereanos. Salud UIS. 2001;33:134-41

23. Prada GE. Percepción social de la alimentación en la zona de intervención del programa CARMEN, Bucaramanga. Reporte técnico. Bucaramanga, Colombia: Universidad Industrial de Santander; 2003.
24. Prada GE, Oróstegui M. Situación alimentaria y nutricional de las madres residentes en un sector rural del municipio de Girón. Salud UIS. 1998;27(1):5-10.

25. Prada GE, Soto A, Herrán OF. Consumo de leguminosas secas en el departamento de Santander, Colombia. 2002-2003. Arch Latinoam Nutr. 2005;55(1):64-70.

26. German JB, Dillard CJ. Saturated fats: what dietary intake? Am J Clin Nutr. 2004;80:550-9.

27. Tanasescu M, Cho E, Manson JE, Hu FB. Dietary fat and cholesterol and the risk of cardiovascular disease among women with type 2 diabetes. Am J Clin Nutr. 2004;79:999-1005.

28. Clifford C, Ballard-Barbash R, Lanza E, Bock G. Diet and cancer risks. Bethesda, Maryland: Nacional Cancer Institute. Disponible en: http://rex.nci.nih.gov/NCI_Pub_Interface/ raterisk/risks73.html. Acceso el 2 de febrero de 2008.

29. República de Colombia, Instituto Colombiano de Bienestar Familiar. Encuesta nacional de la situación nutricional en Colombia, 2005. Capitulo 5. Santa Fe de Bogotá, Colombia: PROFAMILIA, INS, OPS, Universidad de Antioquia; 2006.

30. Herrán OF, Prada GE, Quintero DC. Ingesta usual de vitaminas y minerales en Bucaramanga, Colombia. Rev Chil Nutr. 2007;34(1): 35-44.

31. Carriquiry AL. Estimation of usual intake distributions of nutrients and foods. J Nutr. 2003; $133: 601 s-8 s$.

Manuscrito recibido el 7 de febrero de 2007. Aceptado para publicación, tras revisión, el 20 de diciembre de 2007.

ABSTRACT Objectives. Describe the dietary patterns and extent of access to food among families displaced by armed conflict in a locality of the Santander department of Colombia. Methods. A descriptive study of the food consumption of 101 families living in forced

Dietary patterns and access to food among displaced families in the municipality of Girón, Santander, Colombia

\footnotetext{
Key words Food relief, food supply, risk groups nutrition, diet, Colombia.
}

displacement in the urban and suburban areas of the municipality of Girón, Santander department, Colombia. During the second half of 2003, the person in charge of the family's food preparation completed a sociodemographic survey with questions on the monthly food expenditure, where the food was bought and with what frequency, and a log of food consumption during the preceding 24 hours. The dietary pattern was established according to the frequency of food consumption and preparation through a linear regression model that used the expenditure as a dependent variable.

Results. Of all the families, $34.7 \%$ reported not having access to food shopping and $13.9 \%$ received food donations. The average expenditure on food was 0.52 of the standard minimum wage. The only variable associated with expenditure was the number of family members working and contributing to the family budget $(P=0.037)$; for each working member, expenditure rose by increments of 0.07 of the minimum wage (95\% confidence interval: 0.004- 0.149). No association was found between the length of time of the displacement and the food expenditure. The overall quality of the diet was deficient given that the recommended allowances of fruits, vegetables, and dairy products were not being met.

Conclusions. Food insecurity encompassed $95.0 \%$ of the study families, even though they had been living as refugees for three years. The diet quality was substandard. The principal causes were low household income and a lack of knowledge regarding how to choose nutritionally superior foods. In addition to food donations, relief programs caring for displaced families should provide practical and educational training on nutrition, and thus, successful alternatives. 